\title{
Impact Initiation of Reactive Aluminized Fluorinated Acrylic Nanocomposites
}

\author{
Bradley W. White ${ }^{1}$ Christopher A. Crouse $e^{2} \cdot$ Jonathan E. Spowart $^{3}$. \\ Brady Aydelotte $^{4} \cdot$ Naresh N. Thadhani ${ }^{5}$
}

Received: 4 February 2016/ Accepted: 31 March 2016/Published online: 18 April 2016

(c) Society for Experimental Mechanics, Inc 2016

\begin{abstract}
The initiation of aluminized fluorinated acrylic (AlFA) nanocomposites during modified Taylor impact tests was investigated. Samples were impacted against a steel or sapphire anvil at a nominal velocity of $150 \mathrm{~m} / \mathrm{s}$. A framing camera was used to capture head-on and sideprofile impact images for the sapphire window and steel plate rigid anvils, respectively. Correlations were drawn between both experimental setups to determine the initiation locations and reaction times. Reactions were found to initiate at an intermediate radius on the impact face of the pellet at a time near full compaction. From simulations, the highest strains and temperatures were found at radii similar to those observed in experiments at the time of ignition. Off-normal impacts produced higher localized straining and temperatures on one-half of the pellet. The copper projectile, used for delivery, was revealed to aid in a shear assisted reaction by helping to drive the pellet material outward as the projectile deformed radially.
\end{abstract}

Bradley W. White

white234@1lnl.gov

1 Lawrence Livermore National Laboratory, 7000 East Avenue, Livermore, CA 94550, USA

2 Air Force Research Laboratory, Munitions Directorate, Eglin AFB, FL 32542, USA

3 Air Force Research Laboratory, Materials and Manufacturing Directorate, Wright-Patterson AFB, OH 45433, USA

4 Army Research Laboratory, Aberdeen Proving Ground, MD 21005, USA

5 School of Materials Science and Engineering, Georgia Institute of Technology, 771 Ferst Drive NW, Atlanta, GA 30332, USA
Keywords AlFA - Taylor impact · Polymer composites . Energetic materials $\cdot$ Impact initiation

\section{Introduction}

Polymer-based reactive composites are often studied for use as structural energetic materials with proposed applications as munitions in military operations [1] or solid rocket propellants [2]. These applications often require detailed knowledge of their mechanical and reactive behavior under high-rate loading or impact conditions. In experiments where a pellet of the reactive composite is attached to the front end of a metal projectile and then impacted against a rigid anvil, reactions have been observed [3, 4]. However, the initiating reaction locations within the composite and the time to reaction following the initial impact are not well known in modified Taylor impact tests. This is in part due to observational image data obtained from high-speed cameras being limited to sideprofile angles during the impact process. This obscures the far side of the sample and metal projectile as well as any processes that occur at the impact surface. Furthermore, reactions may initiate within the interior of the samples themselves. This work attempts to address these issues by identifying likely locations of the reaction initiation sites using a modified Taylor impact test geometry.

Depending on the nature of the reaction that occurs during impact, it may be possible to infer what is taking place on the backside of the projectile-composite sample. However, if the reaction appears to be very small in extent or non-existent, then the reaction event may be localized to a certain region of the composite on the far side, or not take place, or not be visible due to limited acquisition capabilities of the camera. To work around these limitations, there 
are options available, such as having mirrors on the opposite side of the camera to allow an image to simultaneously capture both sides of the impact/reaction event. Examining the impact face itself requires an entirely different setup involving a transparent window that can act as a rigid anvil, while also allowing for the head-on impacts to be interrogated [5-7].

In previous work by Crouse et al. [8, 9], an aluminized fluorinated acrylate-based polymer composite (AlFA), reinforced with nano-sized particles, has been shown to be highly reactive over a large range of aluminum particle reinforcement levels. The composite was found to react reliably in both air and partial vacuum at impact velocities greater than $100 \mathrm{~m} / \mathrm{s}$ [9] for various weight percentages (up to $60 \mathrm{wt} \%)$ of nano-aluminum $(80 \mathrm{~nm})$. Thus, this composite system is ideal for the characterization of the reaction initiation characteristics and behavior under impact conditions using a transparent window and steel anvil setups. In this work we chose to use the formulation containing $50 \mathrm{wt} \%$ of nano-aluminum. Additionally, while the modified Taylor impact test is often used as an inexpensive screening tool and determining the relative sensitivity of reactive materials, it also is appropriate for understanding the behavior of AlFA and similarly related energetic composite materials, due to their applications as a munitions casing materials, pyrotechnic indicator materials, and pyrotechnic filament materials [10], where similar loading conditions are likely to occur.

\section{Experimental Methodology}

Oxygen-free high thermal conductivity (OFHC) copper was machined into cylindrical projectiles 7.62 (diameter) $\times$ $30 \mathrm{~mm}$ (length) to act as a delivery mechanism for the composite pellets. The pellets were mounted onto one end of the copper projectiles using a thin layer of epoxy. Care was taken to align the center axis of the pellet with the center axis of the copper projectile. The pellets were nominally $3.0 \mathrm{~mm}$ diameter $\times 1.5$ long and consist of a fluorinated polymer matrix infiltrated with $50 \mathrm{wt} \%$ of nanosized aluminum powder. The amount of porosity for this formulation was measured to be approximately $12 \%$ [8]. Additional details regarding the composite formulation may be found in work by Crouse et al. [8].

The projectiles with the mounted reactive composite pellets were then loaded into a $7.62 \mathrm{~mm} \times 1.8 \mathrm{~m}$ (diameter $\times$ length) Taylor gas gun apparatus as shown in Fig. 1 . Using compressed helium, the projectiles were propelled towards a sapphire window or steel plate anvil at a nominal velocity of $150 \mathrm{~m} / \mathrm{s}$. The sapphire window was $25.4 \mathrm{~mm}$ diameter $\times 50.8 \mathrm{~mm}$ thick and was supported by a plastic buffer and steel cylinder, which has an inner viewing

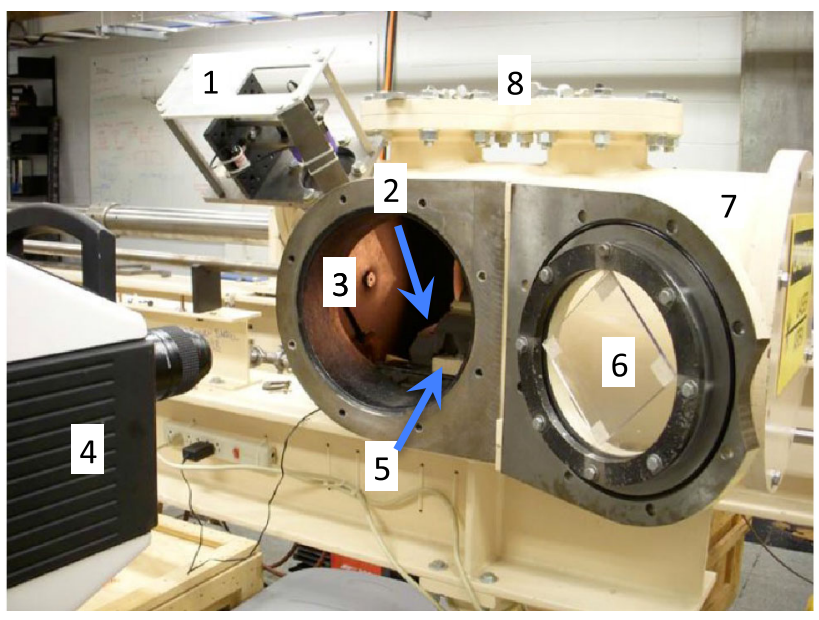

Fig. 1 The $7.62 \mathrm{~mm} \times 1.8 \mathrm{~m}$ Taylor gas gun located at Georgia Institute of Technology. (1) Laser window mount with lasers. (2) Laser detection window. (3) Gun barrel end. (4) Imacon 200 camera. (5) Guide rails for holding the anvil and aid in anvil alignment. (6) Observation port with sacrificial windows for image acquisition. (7) Catch tank end for access to rear of anvil. (8) Secondary ports for gun peripherals

diameter of approximately $20.32 \mathrm{~mm}$. An ultra-high molecular weight polyethylene (UHMWPE) washer located between the steel support cylinder and sapphire window acts as a buffer against stress concentrations. A UHMWPE sleeve was also used to encase the sapphire window. Using this method, the sapphire window was found to survive multiple lower velocity impacts from a copper projectile. The velocity was measured using a twolaser interrupt system in conjunction with an up-down counter (UDC). A schematic of the Taylor gas gun's key components are shown in Fig. 2. In addition to measuring

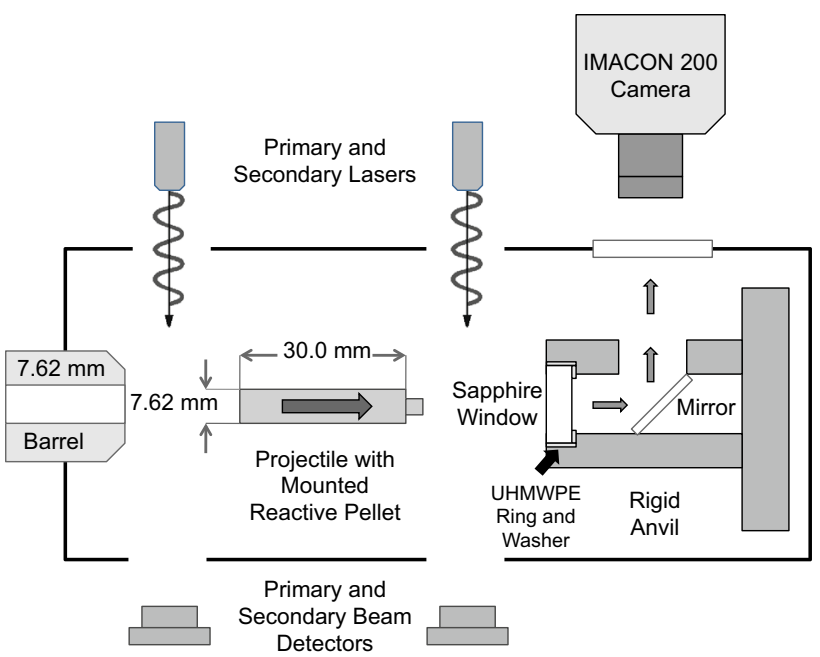

Fig. 2 Schematic of the Taylor gas gun's key components. The rigid anvil in this work was either an S7 steel plate (setup not shown) or sapphire window 
the precise velocity from the triggering of the two laser detectors, the UDC is used to trigger a high-speed camera (Imacon 200) that captures images of the reactive pellet impacting the anvil at specified times. Since the impact occurs within an enclosed chamber the energetic response of the reactive pellets may be captured under ambient or vacuum environmental conditions. Ambient conditions were used for the steel anvil shots and a partial vacuum (145-225 mtorr) was used for the sapphire anvil shots. The partial vacuum in sapphire anvil experiments was used to eliminate contributions of reactions with air as well as help identify reaction initiation sites.

\section{Experimental Results and Discussions}

\section{Impact of Pellets Against a Steel Anvil and Side-Profile Images}

In Figs. 3, 4, and 5, sequences of side-profile images are shown for experiments in which a steel anvil was used. In these images, the projectile with a mounted pellet is shown to impact the anvil, upon which the pellet begins to compact and strain radially. When the pellet appears to be fully compressed, the beginning of a reaction takes place, indicated by the presence of bright regions at the plane of impact at around 7-10 $\mu$ s. Here we define "fully compressed" to be when there is little, to no visible gap between the copper projectile and anvil. This is similar to experiments, by Du et al. [3], involving the compaction of metallic powder mixtures $(\mathrm{Al}+\mathrm{X})$ in which reactions were also found to initiate near full compaction. As this reaction progresses, a mixture of reaction products, unreacted debris, and gas is expelled in a radial direction away from the initial point of impact. Details of the most likely reaction products for this composite system are described in previous work by Crouse et al. [8].

From an examination of images for shot 11138, (Fig. 3) between the initial impact and $\sim 5 \mu$ s $(\sim 8 \mu$ s for shots 11139 and 11140), the pellet deforms extensively. During this time, no indication of media being ejected is visible. This may be due to the high polymer matrix content allowing for a large amount of strain to accumulate without undergoing extensive fracturing and fragmentation. Between 5 and $7 \mu \mathrm{s}(8-10 \mu$ s for shots 11139 and 11140), the pellet becomes "fully" compressed between the copper projectile and anvil. During this period, the polymer matrix and nano-aluminum particles are strained even further between the copper projectile and anvil. At approximately $7 \mu \mathrm{s}$, comminution of the pellet has begun with fragments ejected outward. Due to the large increase in surface area of the nano-aluminum and strain-induced mixing with the fluorinated polymer matrix, and with oxygen present in the surrounding environment a reaction initiates. This is
Fig. 3 Impact images for shot 11138 , in ambient air. The projectile impact velocity and vertical tilt were measure to be $145.1 \mathrm{~m} / \mathrm{s}$ and $0.9 \pm 0.25^{\circ}$ respectively. The time to first observable reaction occurred at $7 \mu$ s and is visible across the entire impact surface

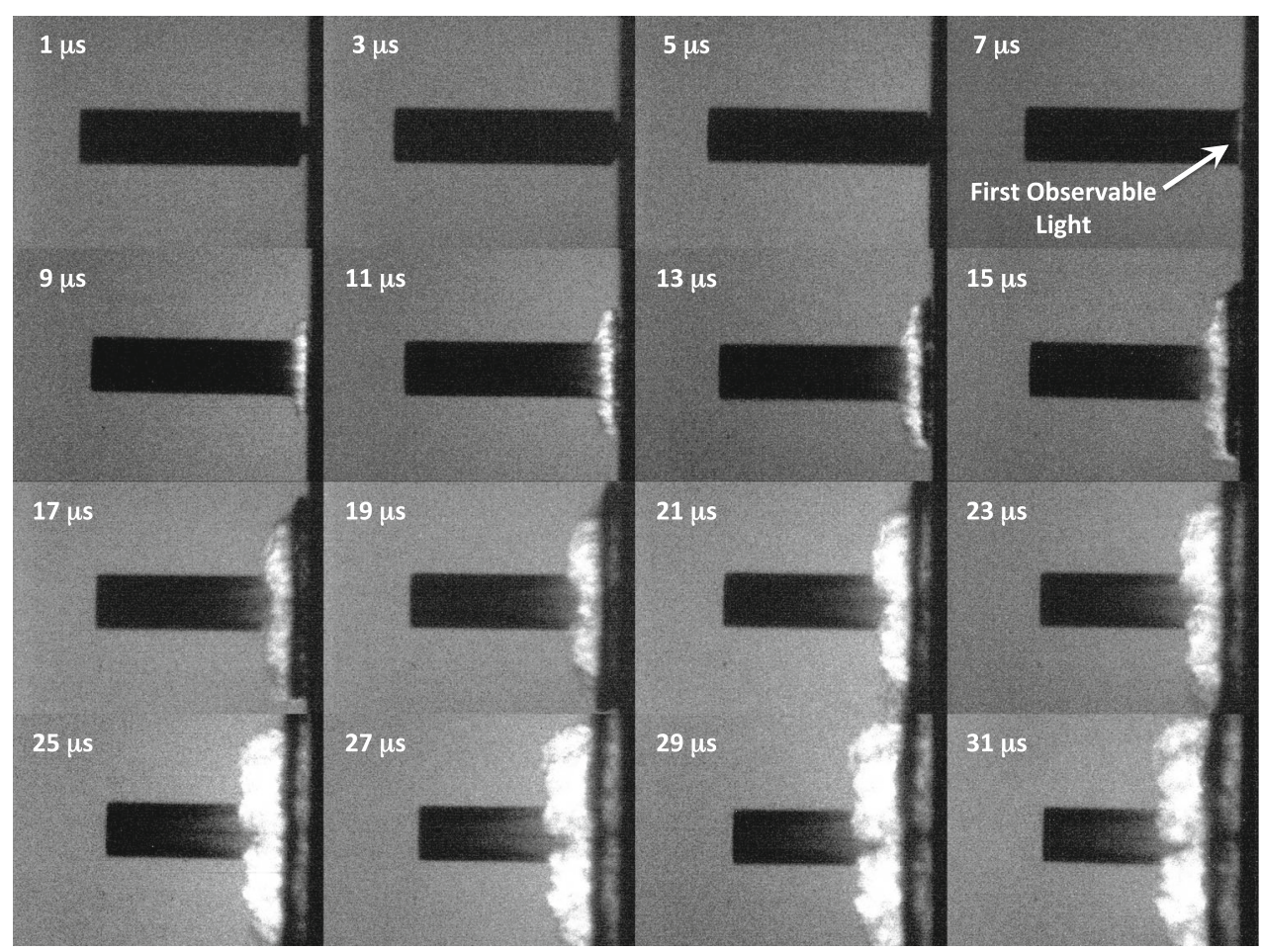


Fig. 4 Impact images for shot 11139 in ambient air. The projectile impact velocity and vertical tilt were measure to be $146.2 \mathrm{~m} / \mathrm{s}$ and $1.26 \pm 0.25^{\circ}$ respectively. The time to first observable reaction occurred at $10 \mu$ s and is visible near the top portion of the impact surface

Fig. 5 Impact images for shot 11140 in ambient air. The projectile impact velocity and vertical tilt were measure to be $147.1 \mathrm{~m} / \mathrm{s}$ and $0.12 \pm 0.25^{\circ}$ respectively. The time to first observable reaction occurred at $10 \mu$ s and is visible across the entire impact surface
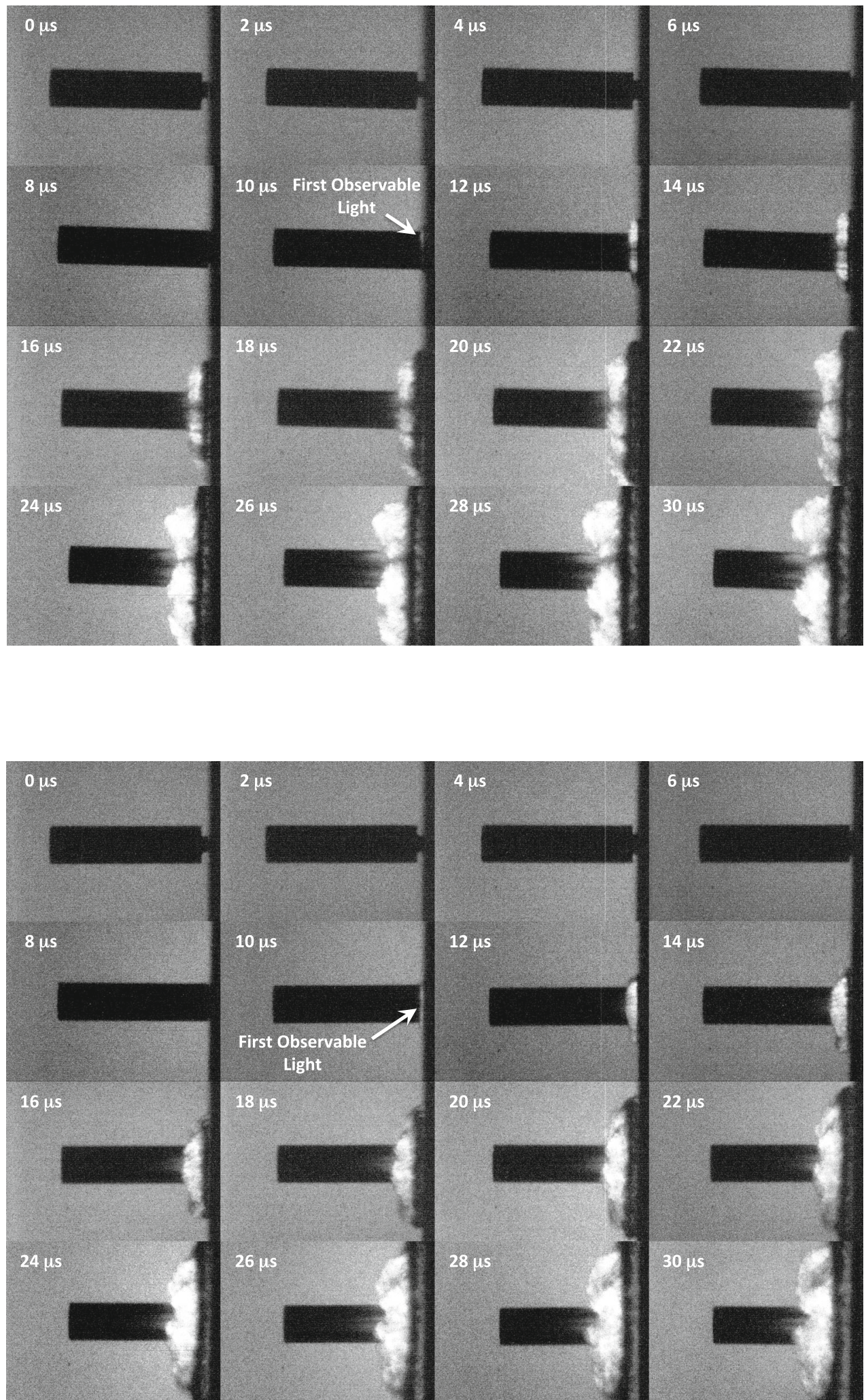
indicated by the presence of light in Figs. 3, 4, and 5, at 7, 10 , and $10 \mu \mathrm{s}$, respectively. From the side profile images it is unclear if comminution occurs prior to or is induced by the reaction within the pellet.

At times greater than $10 \mu \mathrm{s}$, the unreacted composite material continues to be loaded between the copper projectile and anvil before slowing down and rebounding. Throughout the entire captured duration of each shot, the back end of copper projectile continued to move towards the anvil and had not yet transitioned from a loaded to unloaded (rebounded) state. At these later times the reaction cloud obscures the impact end of the copper projectile. However, from our experience with inert experiments and simulations (without a reactive pellet) the copper projectile plastically deforms at the impact face for approximately 30 $\mu$ s following impacts at $\sim 150 \mathrm{~m} / \mathrm{s}$ and with the geometry used in this work. The overall projectile deformation occurs over a longer period and corresponds to transient times greater than those captured with the imaging acquisition rates chosen for these experiments. This is further evidenced by the continued translation of the projectiles back-end towards the anvil. This continued projectile deformation will cause further mixing and deformation of the nano-aluminum thereby promoting heat generation through plastic work and increase the propensity for maintaining reactions with faster reaction rates. Moreover, since polymer matrix composites are inherently heterogeneous by their nature, localized increases in temperature will develop at the particle scale during dynamic loading events. This can also contribute to enhanced reactions during the compaction and loading processes.

In the images for times greater than $7 \mu \mathrm{s}$, in shot 11138 and $10 \mu$ s in shots 11139 and 11140 , a cloud of hot gas is observed to billow perpendicularly outward from the anvil.
For shot 11139, which impacted at the largest tilt angle $\left(1.26^{\circ}\right)$, the cloud appears to be less uniform, indicating that impact angle may have an important role in reaction events. Nearer to the anvil there is also an appearance of a dark ring, which may be either previously reacted material (carbonaceous byproducts of the fluorination reaction [8]) or unreacted debris from early pellet fragmentation. However, there is a minimal amount of the dark ring visible at earlier times, and it does not appear to be less dense at later times. This, along with a presence of light between the darker ring and anvil face, suggests that the darker ring is primarily comprised of previously reacted material that is propelled outward by the expansion of hot gas. The darker ring appears to expand radially outward at a higher rate than the main cloud. This may be due to an increase in gas pressure generated by reactions taking place in the main cloud, and a partial confinement effect created by the steel anvil.

Using the diameter of the projectiles in Figs. 3, 4, and 5 as a reference scale for each image, an estimate of the main gas cloud thickness and radial distance from the anvil/ projectile were obtained for each shot. Similarly, the radial distance of the darker ring from the projectile was measured. For this analysis, the reference point on the projectile for cloud diameter measurements was located as near to the reacting cloud as possible. The results of the radial distances for times following the initial observed reaction are shown in Fig. 6(left) and 6(right) for the main cloud and dark ring, respectively. The main cloud and dark ring distance profiles for each shot are very similar to one another, indicating that, at a nominal impact velocity of $150 \mathrm{~m} / \mathrm{s}$, their reaction mechanisms are dominated by the same processes, despite slight differences in their initial reaction times. Because of their similar time profiles, the
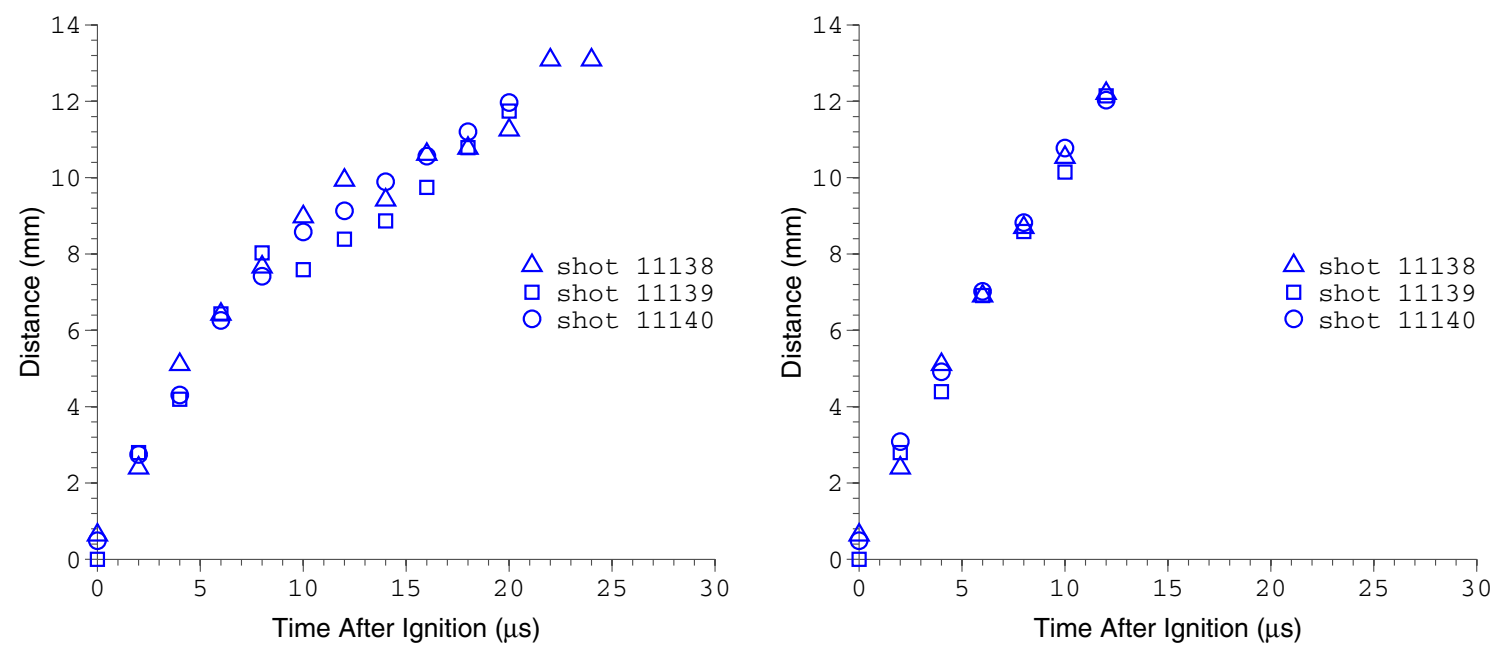

Fig. 6 Main cloud $(l e f t)$ and dark ring $(r i g h t)$ radial distances measured from outer projectile radius. The times have been shifted so that $t=0 \mu$, is the first frame with an observable reaction. The error in distance measurements is estimated to be $0.2 \mathrm{~mm}$ 
distances were averaged over all three shots (see Fig. 7). While initially the main cloud and dark ring have similar slopes, the dark ring continues to progress at a relatively constant velocity of $975 \mathrm{~m} / \mathrm{s}$, whereas the main cloud slows down to approximately $366 \mathrm{~m} / \mathrm{s}$. These values are similar to the deflagration velocities found by Dolgoborodov et al. [11] for confined linear burn tests conducted on aluminum

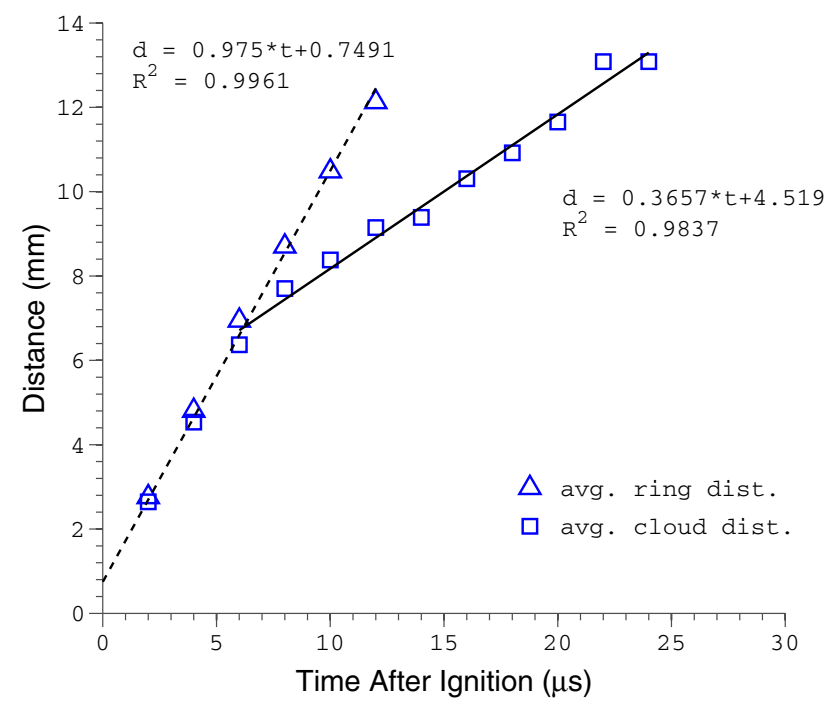

Fig. 7 Averaged radial distances (over all three shots) for the main cloud and dark ring. Linear fits to the data were used to obtain velocities reported in main text. The error in distance measurements is estimated to be $0.2 \mathrm{~mm}$
+ Teflon mixtures. Depending on the mixture's composition, it was found that the steady-state propagation velocities range from 700 to $1300 \mathrm{~m} / \mathrm{s}$. The main cloud thicknesses as a function of time following the reaction are approximately equal to the measured main cloud radial distances.

\section{Impact of Pellets Against a Sapphire Anvil and Head-On Images}

For the sapphire anvil shots, which were used to examine the reaction head-on, the ignition times were found to be similar to those observed in the side profile images. Two shots were conducted with almost identical impact velocities, shot 11211 at $152.9 \mathrm{~m} / \mathrm{s}$ and shot 11212 at $152.6 \mathrm{~m} / \mathrm{s}$. Shot 11211 ignited between 8 and $10 \mu$ s following impact and shot 11212 at approximately $7.5 \mu$ s (see Figs. 8, 9).

The results from side profile investigations suggest that the reaction appears to initiate nearly upon full compaction of the pellet. However, any view of the ignition location is obscured. From head-on images (Figs. 8, 9), particularly for shot 11212, the ignition is seen to emanate at an intermediate radius within the compacted nano-composite. A contrast and brightness enhanced frame image from shot 11212 showed multiple initiation sites that were arranged in a crescent shape (see Fig. 10). At later times additional initiation sites were observed in the frame corresponding to $8.75 \mu$ s following impact that appear to be distinctly disjoint from the nearby reacting material. At $10 \mu$ s for shot
Fig. 8 Shot 11211. Impact velocity of $152.9 \mathrm{~m} / \mathrm{s}$

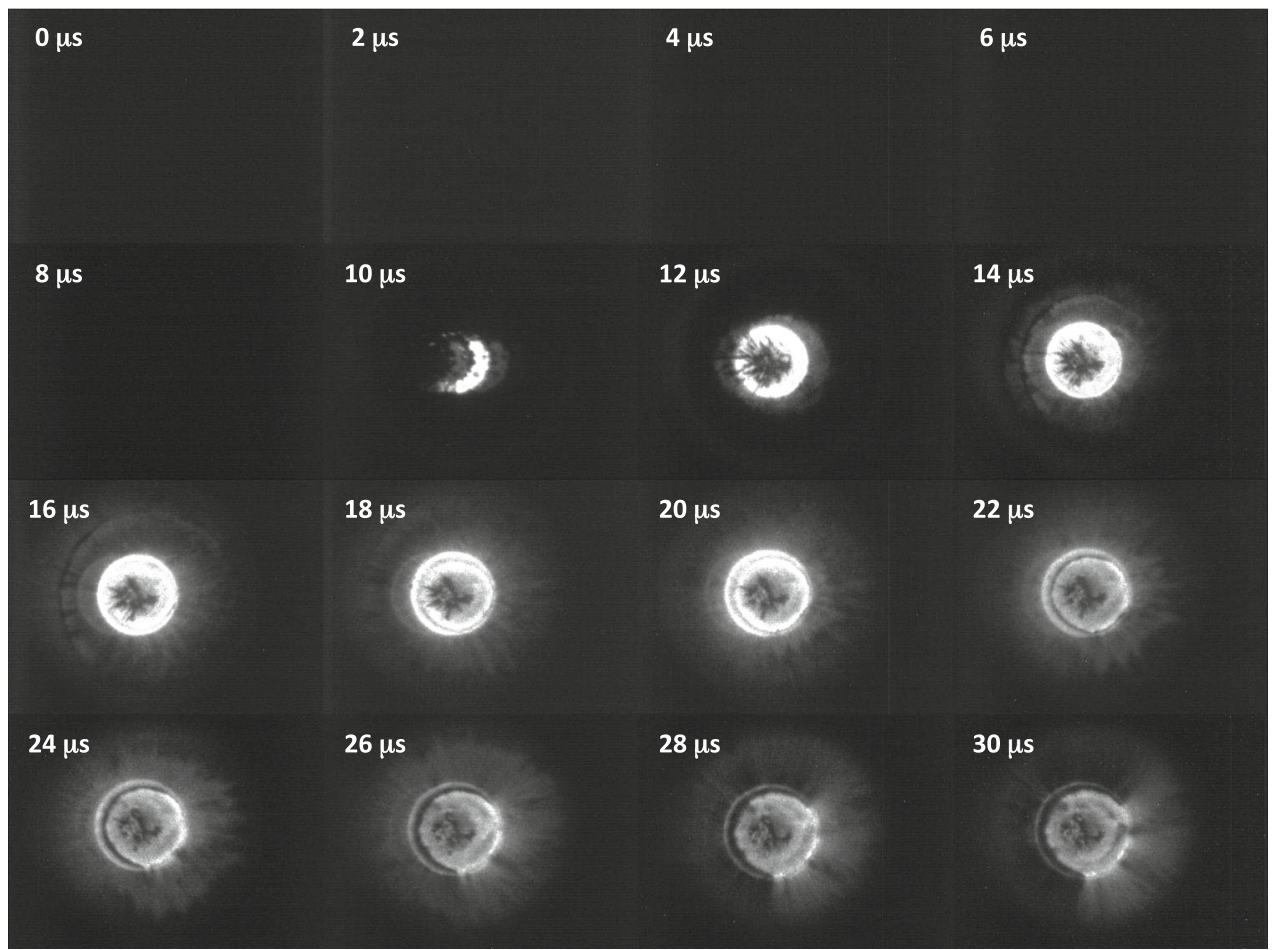


Fig. 9 Shot 11212. Impact velocity of $152.6 \mathrm{~m} / \mathrm{s}$. Camera timings were slightly different for this shot to capture more frames at earlier times following impact

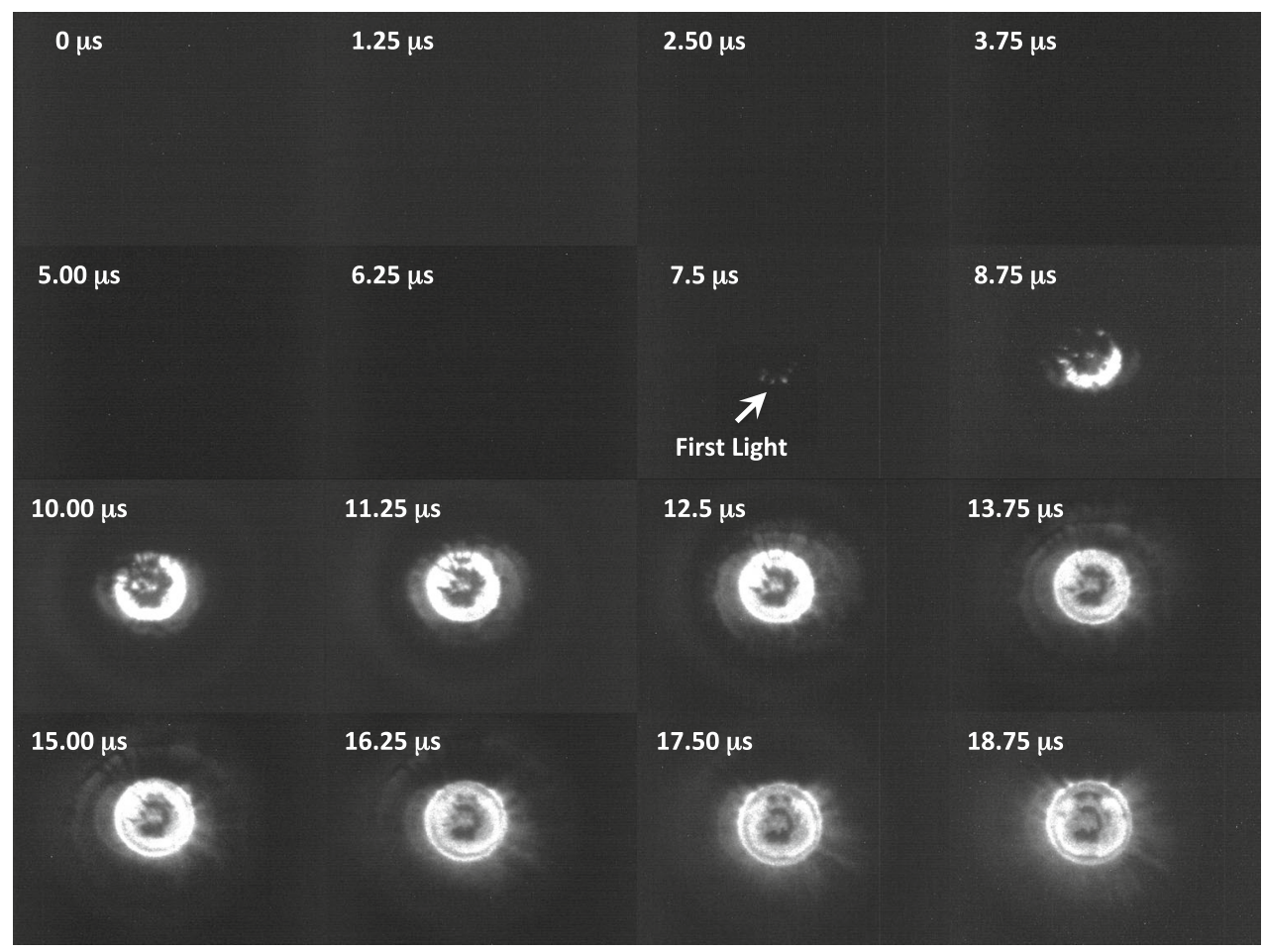

11211 and $8.75 \mu$ s for shot 11212 there are similar crescent-shaped reacting regions. In comparison to side profile impacts this is believed to correspond to similar extents of reaction observed in Fig. 4 (shot 11139) at a time of $10 \mu \mathrm{s}$, which has a more pronounced state of reaction near the top of the impacted region but has not yet fully evolved to enclose the whole perimeter of the compacted pellet. The crescent shaped regions were also observed to have variability in the smoothness of their perimeters (see Figs. 8, 9

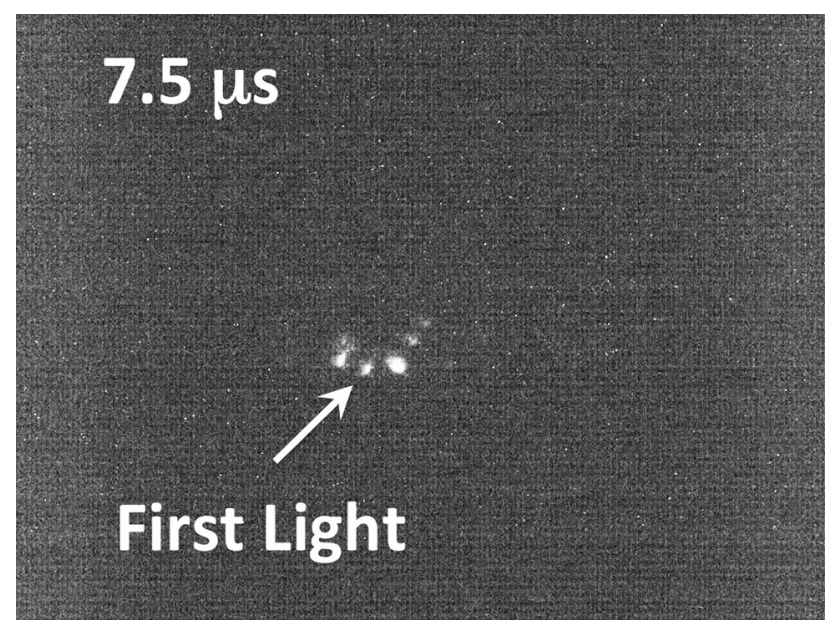

Fig. 10 Enhanced image frame, showing the first observed light for shot 11212. Multiple initiation sites are visible, which are arranged in a crescent shape. At later times these sites coalesced into a single propagating reaction zone at 10 and $8.75 \mu \mathrm{s}$, respectively). This may be due to heterogeneity of the composites leading to heterogeneous initiation.

After initiation, the reaction progresses until a highly uniform circular region is burning (within 2-3 $\mu$ s). This circular region increases in diameter slightly, and the reaction is sustained while under loading. Also of note is the presence of a darker region near the center of the circle. This darker interior region is relatively large initially and decreases size as material continues to be consumed by the reaction. The reaction itself burns with greatest intensity/ brightness for approximately the first $10 \mu \mathrm{s}$, after which it decreases. This is in contrast to the side-profile images that show the reaction burning most intensely at the later times. This suggests the material burning with greatest intensity in the head on images is continuing to react while jetting outward. While doing so, it contributes to the most intense stages of the reaction seen in the side-profile images. The jetting of the material is prevalent throughout the entire reaction, with occasional gaps as seen in the images. This may lead to some of the cloud variability observed in the side-profile images.

One surprising observation is the lack of more intense, bright jets radiating away from the reacting center. Beyond the extent of the circular reacting region the brightness decreases quickly. This is most likely related to the dark ring observed in the side-profile images that we suspect is previously reacted material that is propelled outward. 
Reactions are clearly taking place away from the main impact location (i.e. the billowing cloud observed in sideprofile images) as well as the within the circular region. Depending on the availability of environmental oxygen and/or fluorine from the polymer matrix that can react with the nano-aluminum, either a fluorination or oxidation reaction is most likely to occur.

The footprint of the reacting region increases only slightly in diameter within the measured time. Shown in Fig. 11, is an outline of the approximate viewing area visible from the backside of the sapphire window. This outline is determined from the halo/brighter region surrounding the main reacting material in the center. This is most easily visible in Fig. 8 for the frames corresponding to 26,28 , and $30 \mu \mathrm{s}$ after the initial impact. Using the known visible window size $(20.32 \mathrm{~mm})$ as a scaling factor, measurements were taken of the reacting footprint's size for shot 11211. The footprint increased in diameter from $7.5 \pm 0.5$ to $8.65 \pm 0.5 \mathrm{~mm}$ between 12 and $16 \mu \mathrm{s}$ at a rate of $288 \mathrm{~m} / \mathrm{s}$ and at a rate of $144 \mathrm{~m} / \mathrm{s}$ between 16 and $22 \mu \mathrm{s}$ $(9.52 \pm 0.5 \mathrm{~mm})$. The final observable diameter for this shot was $9.8 \pm 0.5 \mathrm{~mm}$, giving a rate of expansion for the footprint of approximately $35 \mathrm{~m} / \mathrm{s}$. From the individual frames the reaction light had the highest intensity during the period of $12-22 \mu \mathrm{s}$, correlating with the duration of highest rates of the footprint's expansion.

\section{Computational Studies of Impact Ignition in Modified Taylor Impact Tests}

\section{Modeling Setup}

To model the early impact stages of high deformation that lead up to initiation of the composite, we implemented the

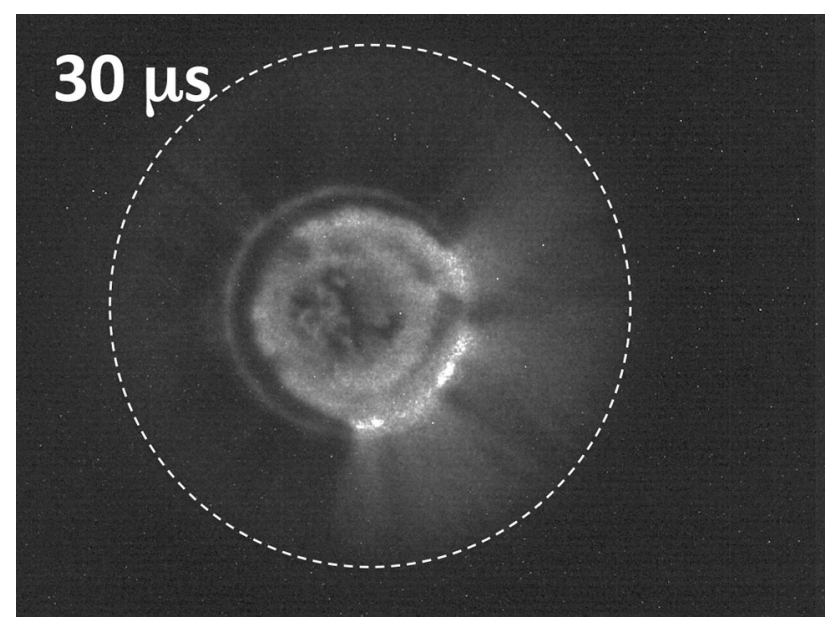

Fig. 11 Subsample of shot 11211 at a time of $30 \mu$ s following the initial impact with the approximate boundary of the sapphire anvil's viewing region (20.32 $\mathrm{mm}$ diameter)
Zerilli-Armstrong constitutive model [12, 13], which has been used for PTFE [12-14] and composites with PTFE and $\mathrm{Al}$ [15], and is well suited for describing the behavior of strain hardening polymeric materials at high rates of strain. For modeling purposes we chose to use PTFE as a representative matrix material since limited strength data exists for the fluorinated acrylate matrix and AlFA composites. While it would be ideal to have constitutive models describing the behavior of the fluorinated acrylate (FA) used in this work, the quasi-static strengths for PTFE (10$20 \mathrm{MPa})[14,16]$ and FA ( $<15 \mathrm{MPa})[8]$ for a strain rate of $\sim 0.001 \mathrm{~s}^{-1}$ are fairly close. The acrylate-containing polymer, PMMA, was also considered. However, PMMA has a much higher quasi-static compressive strength that is greater than $70 \mathrm{MPa}[17,18]$ for the same strain rate.

For a composite comprised of 26.5/73.5 wt $\%$ of $\mathrm{Al}$ and PTFE, Casem et al. applied the Zerilli-Armstrong (Z-A) model to experimental data for uniaxial compression

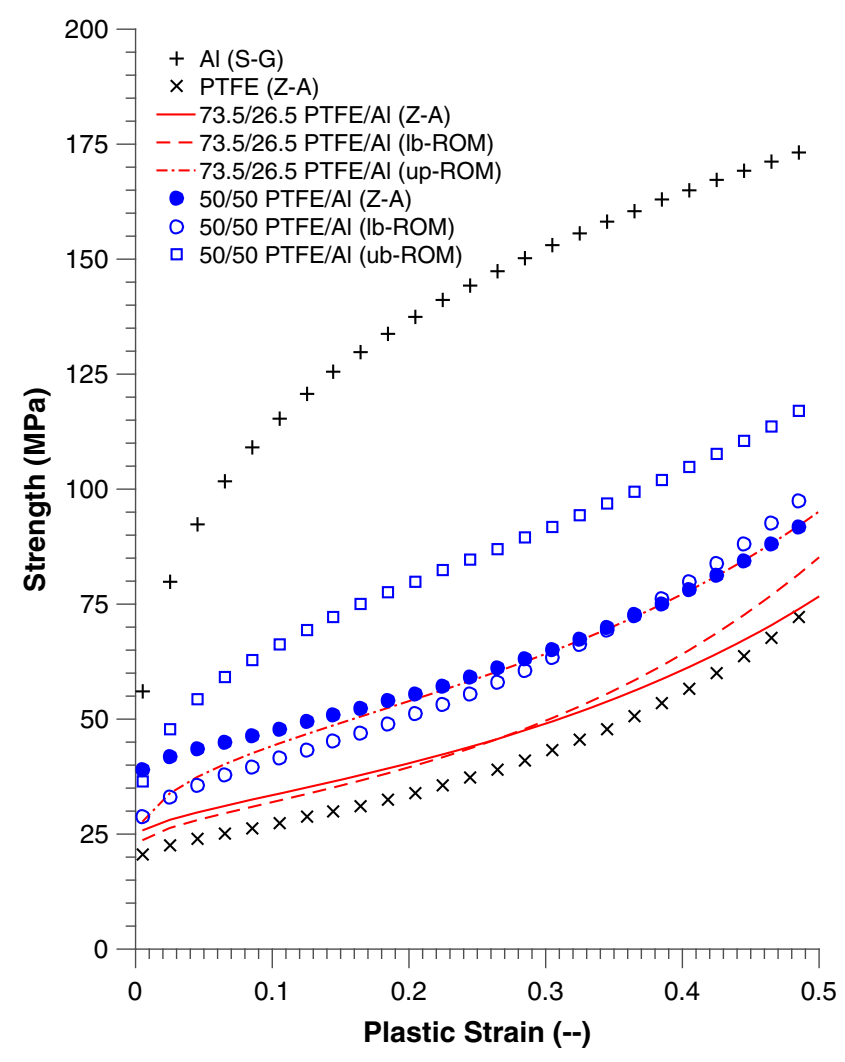

Fig. 12 The modeled constitutive behavior of the constituents (black), composite containing 73.5/26.5 wt\% PTFE/Al (red), and composite containing 50.0/50.0 wt\% PTFE/Al (blue). Using the Zerilli-Armstrong (ZA) model parameters determined by Casem et al. the behavior of the composite containing 73.5/26.5 wt\% (19.65/80.35 vol.\%) PTFE/Al was found to lie more closely to the lower bound rule of mixtures. The ZA model parameters for composite containing 50.0/ $50.0 \mathrm{wt} \%$ PTFE/Al were modified to have a response similar to the lower bound rule of mixtures for that formulation (Color figure online) 
experiments at a strain rate of $2500 \mathrm{~s}^{-1}$, and obtained fair agreement between the experiments and modeling [15]. We compared this composite's response to the SteinbergGuinan (S-G) strength model for pure aluminum [19] and Zerilli-Armstrong model for pure PTFE. We found the strength behavior of the composite of Casem et al. to have a more similar response to that of PTFE than aluminum at a strain rate of $2500 \mathrm{~s}^{-1}$. This can be seen in Fig. 12, which shows the stress-strain response of each material. This observation is logical, based on the relative proportions of PTFE and Al in this material. To further understand the behavior of the types of composites in this study we also used the following lower- [20] and upper-bound [21] rule of mixtures relationships shown in Eqs. 1 and 2, respectively, to determine the composite stress for the 26.5/73.5 wt\% Al/PTFE composite. We then compared the result with the response found by Casem et al. [15] using the Zerilli-Armstrong model.

$$
\begin{aligned}
& \left\langle\frac{1}{X}\right\rangle=\sum_{i=1}^{N} \frac{\phi_{i}}{X_{i}} \\
& <X>=\sum_{i=1}^{N} \phi_{i} X_{i}
\end{aligned}
$$

Here, $\phi_{i}$ and $X_{i}$ are the volume fraction and stress (determined from either the S-G model for Al or Z-A model for PTFE) at a particular overall composite strain level, $e$, of each constituent, $i$. These bounds on the mechanical behavior of the composite are plotted along-side those of pure aluminum and PTFE in Fig. 12. In this figure the stress vs. plastic strain response of the composite with 26.5/ $73.5 \mathrm{wt} \%$ Al/PTFE more closely follows the response predicted by the lower-bound mixture. Thus, we applied the same approach for a composite containing 50/50 wt $\%$ $\mathrm{Al} / \mathrm{PTFE}$ using the lower bound response as an idealized reference high-rate stress-strain response for the surrogate AlFA composite. We then used the reference curve to calibrate a new set of Z-A model parameters (see Fig. 12) at a strain-rate of $2500 \mathrm{~s}^{-1}$, which were then used in our simulations. Due to the fairly small sample size used in the modified Taylor impact tests we expect the strain-rates to vary during impact and be no larger than one or two orders of magnitude larger. The $\mathrm{Z}-\mathrm{A}$ model has strain rate dependency built into the model, which for higher strainrates results in a shift of the stress-strain response of the AlFA composite upwards. For constant strain-rates of 25,000 and $250,000 \mathrm{~s}^{-1}$ the initial strength is calculated to increase from $39 \mathrm{MPa}$, at a strain of $2500 \mathrm{~s}^{-1}$, to 50 and 64 $\mathrm{MPa}$, respectively. The $\mathrm{Z}-\mathrm{A}$ model parameter values for each Al/PTFE composite, and $100 \mathrm{wt} \%$ PTFE are listed in Table 1 and the S-G strength model parameters used for aluminum are listed in Table 2. For the Grüneisen equation-of-state of each material a seven-term polynomial is used [22], in which the pressure $(P)$ is related to the compression $(\mu)$ and energy per reference volume $(E)$ of the material through the relation:

Table 1 Zerilli-Armstrong strength parameters values for PTFE [13], 73.5/26.5 wt\% PTFE/Al [15], and 50.0/50.0 wt\% PTFE/Al

\begin{tabular}{lllll}
\hline $\begin{array}{l}\text { Model } \\
\text { Parameter }\end{array}$ & PTFE & $\begin{array}{l}73.5 / 26.5 \mathrm{wt} \% \\
\text { PTFE/Al }\end{array}$ & $\begin{array}{l}50.0 / 50.0 \mathrm{wt} \% \\
\text { PTFE/Al }\end{array}$ & $\begin{array}{l}\text { Parameter } \\
\text { Units }\end{array}$ \\
\hline$\beta_{0}$ & 0.0201 & 0.011672 & 0.012 & $1 / \mathrm{K}$ \\
$\beta_{1}$ & 0.000264 & 0.000139 & 0.00037 & $1 / \mathrm{K}$ \\
$\alpha_{0}$ & 0.00478 & 0.0 & 0.00478 & $1 / \mathrm{K}$ \\
$\alpha_{1}$ & 0.0000502 & 0.0 & 0.0000502 & $1 / \mathrm{K}$ \\
$\omega_{0}$ & -3.6 & -3.0 & -3.0 & - \\
$\omega_{1}$ & -0.625 & -0.5 & -0.5 & - \\
$\omega_{p}$ & -40.0 & 0.0 & -31.0 & $1 / \mathrm{GPa}$ \\
$B_{p a}$ & 4.016 & 0.55 & 0.55 & 20.0 \\
$B_{p b}$ & 20.0 & 0.0 & 0.714 & $1 / \mathrm{GPa}$ \\
$B_{p n}$ & 0.714 & 0.0 & 0.1 & $1 / \mathrm{GPa}$ \\
$B_{0 p a}$ & 0.0724 & 0.025 & 22.0 & - \\
$B_{0 p b}$ & 22.0 & 0.0 & 0.5 & $1 / \mathrm{GPa}$ \\
$B_{0 p n}$ & 0.5 & 0.0 & & $1 / \mathrm{GPa}$ \\
\hline
\end{tabular}

Table 2 Steinberg-Guinan strength parameters values for aluminum (1100-O) [19]

\begin{tabular}{llllll}
\hline $\begin{array}{l}A \\
(1 / \mathrm{GPa})\end{array}$ & $\begin{array}{l}B \\
(1 / \mathrm{K})\end{array}$ & $\begin{array}{l}G_{0} \\
(\mathrm{GPa})\end{array}$ & $\begin{array}{l}Y_{0} \\
(\mathrm{GPa})\end{array}$ & $\begin{array}{l}n \\
(-)\end{array}$ & $\begin{array}{l}\beta \\
(-)\end{array}$ \\
\hline 0.0652 & 0.00616 & 27.1 & 0.04 & 0.27 & 400 \\
\hline
\end{tabular}


$P=a_{0}+a_{1} \mu+a_{2} \mu^{2}+a_{3} \mu^{3}+\left(b_{0}+b_{1} \mu+b_{2} \mu^{2}\right) E$

where $a_{0}$ is a pressure constant, $a_{1}, a_{2}$, and $a_{3}$ the bulk moduli coefficients, $b_{0}$ the Grüneisen coefficient, and $b_{1}$ and $b_{2}$ corrections to the Grüneisen coefficient. However, in the present work, $a_{0}, b_{1}$, and $b_{2}$ were set to zero, simplifying Eq. 3.

$P=a_{1} \mu+a_{2} \mu^{2}+a_{3} \mu^{3}+b_{0} E$

Using the same approach implemented for the constitutive behavior we defined a set of elastic and equation of state model parameters to create a full set of parameters that describe a surrogate composite material. This is used to interrogate the impact initiation mechanisms of an AlFA composite containing $50 \mathrm{wt} \%$ of nano-sized aluminum particles. The equation-of-state and elastic parameters for each material are given in Table 3 along with their elastic and shear moduli.

These parameters were implemented in three dimensional Lagrangian simulations using the arbitrary Lagrangian-Eulerian hydrocode, ALE3D [23], to examine the state of the composite pellet at initiation times found in modified Taylor impact experiments. The copper projectile and composite pellet had the same geometry as experiments, and an impact velocity of $146 \mathrm{~m} / \mathrm{s}$. Simulations varied the impact angle up to five degrees, which exceeds the observed range of angles, found in side-profile experimental images and included the use of a friction coefficient of 0.2 between the anvil face, pellet, and projectile. The anvil's constitutive behavior was modeled using the Steinberg-Guinan strength model with parameters for $4340 \mathrm{Rc}$ 38 steel [19]. Figure 13 shows the initialization of a simulation with an impact tilt angle of two degrees. The axial and radial mesh sizes used in the series of calculations were 15 and 30 zones $/ \mathrm{mm}$, respectively. While, pellet fracturing and post-reaction effects may be important, simulations did not include any failure or chemistry models.

\section{Modeling Results and Discussions}

In Fig. 14, the impact face of three-dimensional simulations are shown for impacts with tilt angles of $0,0.5^{\circ}, 1^{\circ}$,

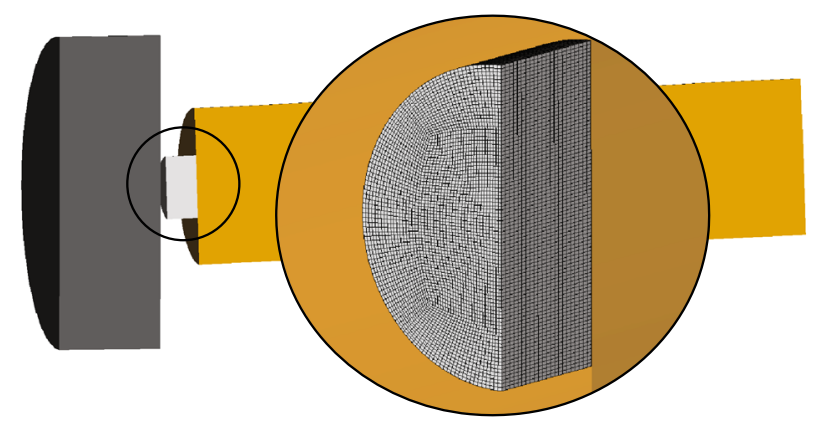

Fig. 13 Initial setup of half-symmetry three-dimensional simulations and pellet mesh for an impact angle of $2^{\circ}$

$2^{\circ}$, and $4^{\circ}$ and at 6 (left) and $8 \mu$ s (right) following impact against the rigid steel anvil. The temperature and plastic strain are plotted on the left- and right-hand sides of each subfigure, respectively. Throughout this elapsed duration the diameter of the pellet did not strain beyond the diameter of the copper projectile (outlined in Fig. 14). At earlier times $(<6 \mu \mathrm{s})$, the regions of highest plastic strain and temperature were located in the interior and rear of the pellet. Between 6 and $8 \mu$ s these regions moved forward, towards the impact face of the sample, with increasing velocity as the impact angle was increased. At impact durations later than $9.4 \mu \mathrm{s}$, the pellet strained beyond the copper projectile and the simulations became unstable. Results are not reported for these final stages of the simulations.

During a normal impact angle initiation event, the regions of highest strain and temperature were located in a narrow ring on the impact face at an intermediate radius. As the tilt angle is increased the region is more concentrated towards the side of the pellet that first impacts the anvil. Rather than taking on a ring appearance, as shown for the zero to very small tilt angle cases, the footprint of this region is crescent-shaped and resembles that of the initial reaction zones in the sapphire anvil experiments. At $6 \mu$ s, (see Figs. 8, 9) the simulated increase in temperature $(\sim 416 \mathrm{~K})$ is higher than the reported decomposition temperature of $357 \mathrm{~K}$ for the fluorinated acrylate [8]. By 8 $\mu$ s the maximum temperature increased to $518 \mathrm{~K}$, however there were only slight differences in the maximum observed temperatures with changes in impact angle. These
Table 3 Elastic and seven-term polynomial EOS model parameters for polymer binders

\begin{tabular}{llllllll}
\hline Materials & $\begin{array}{l}\rho \\
\left(\mathrm{g} / \mathrm{cm}^{3}\right)\end{array}$ & $\begin{array}{l}\mathrm{E} \\
(\mathrm{GPa})\end{array}$ & $\begin{array}{l}\mathrm{G} \\
(\mathrm{GPa})\end{array}$ & $\begin{array}{l}a_{1} \\
(\mathrm{GPa})\end{array}$ & $\begin{array}{l}a_{2} \\
(\mathrm{GPa})\end{array}$ & $\begin{array}{l}a_{3} \\
(\mathrm{GPa})\end{array}$ & $\begin{array}{l}b_{0} \\
(-)\end{array}$ \\
\hline PTFE & 2.15 & 1.02 & 0.36 & 1.5 & 0.5 & 35.0 & 0.59 \\
26.5/73.5 wt\% Al/PTFE & 2.29 & 1.26 & 0.45 & 1.2 & 0.36 & 68.0 & 0.86 \\
$50 / 50 \mathrm{wt} \% \mathrm{Al} / \mathrm{PTFE}$ & 2.40 & 1.81 & 0.64 & 1.53 & 0.005 & 71.0 & 1.20 \\
\hline
\end{tabular}

The EOS $a_{1}, b_{1}$, and $b_{2}$ were all set to zero. Parameters for PTFE were taken from White and Springer [24] 
(a)
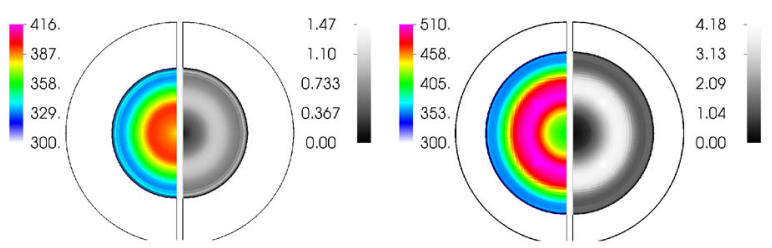

(b)
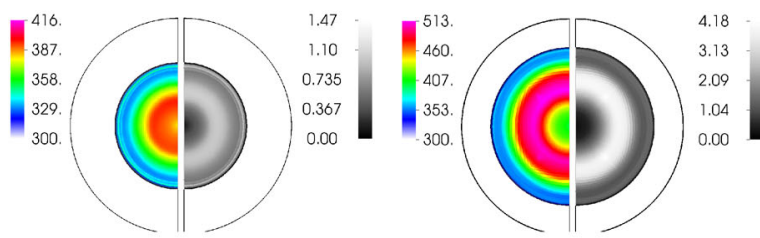

(c)

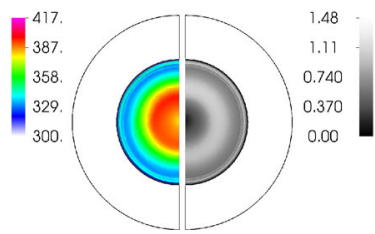

(d)
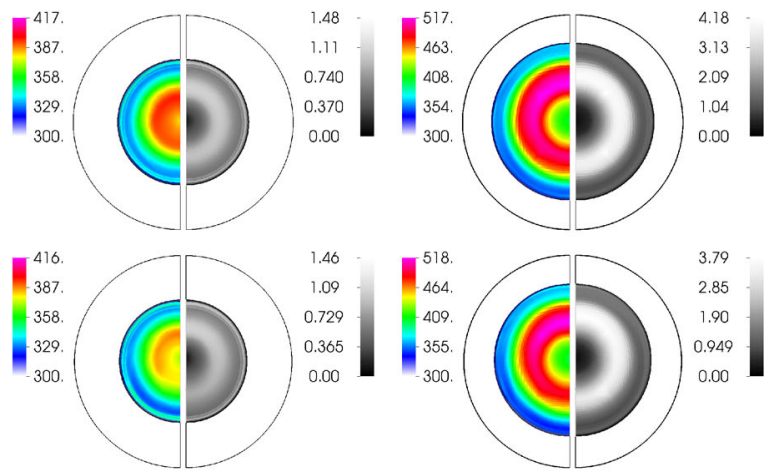

(e)
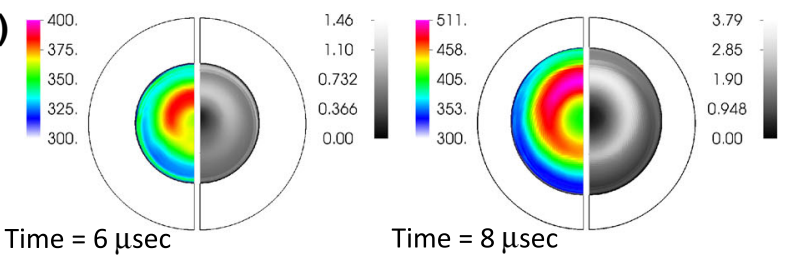

Fig. 14 The composite temperature (in Kelvin, left half) and plastic strain contours (right half) at the impact face for tilt angles of $0.0^{\circ}$ (a) $0.5^{\circ}(\mathbf{b}) 1.0^{\circ}$ (c) $2.0^{\circ}(\mathbf{d})$ and $4.0^{\circ}(\mathbf{e})$ at approximately 6 (left) and 8 $\mu$ s (right) after initial impact. Tilt angle had a large effect on the location of regions with highest strain and temperature

small differences in temperature (and strain) are due to the time of initial impact being chosen as the time for which any portion of the pellet comes into contact with the anvil. If however, an impact time is chosen to be when the center of the pellet face first comes into contact with the anvil, then the maximum observed temperatures (and strain) increase substantially with increasing tilt angle.

These regions of high strain and high temperature are the result of shearing along a band that originates near the center of the pellet's rear face and extends towards the front face, at an angle that steepens with time. Contributing to the formation and progression of the shear band is the deformation of the copper projectile around the pellet, which forms an embedded cup, as shown in Fig. 15. These results are similar to those shown in work by others $[3,4]$ that investigated the impact initiation and compaction of pellets made of energetic metallic powder mixtures $(\mathrm{Al}+$ $\mathrm{X}$ ) with a similar experimental setup, although at a much higher impact velocities.

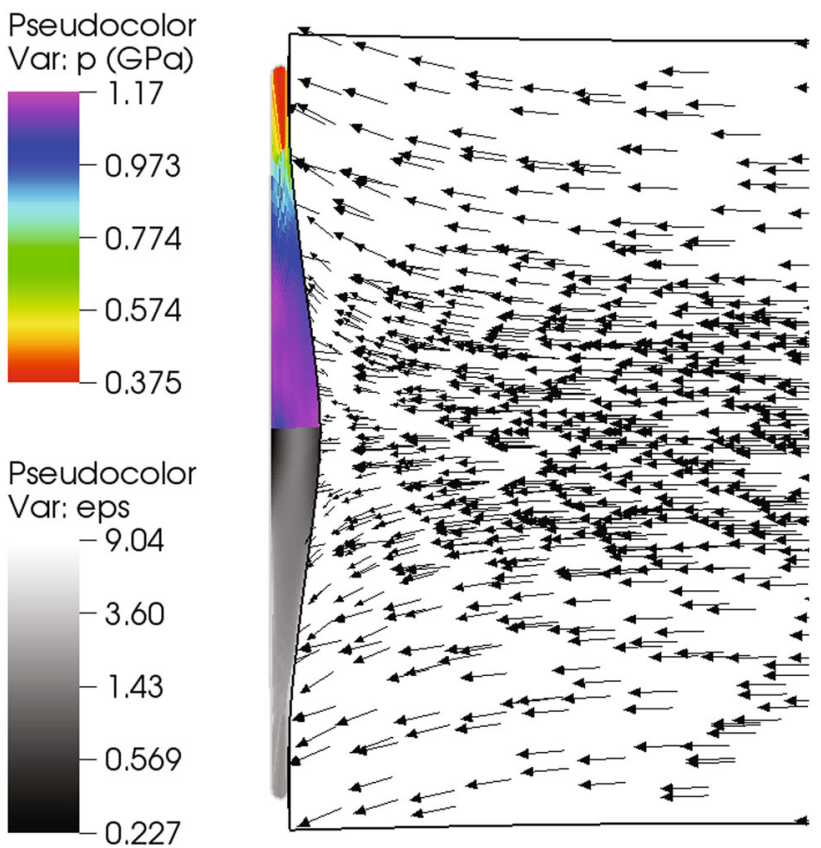

Fig. 15 Velocity vector field within the projectile along with the pressure (top half) and plastic strain (bottom half) contours within the pellet $9.4 \mu$ s after impact with a rigid anvil at a normal incident angle

In Fig. 15, a cross-sectional view of the velocity vector field for the projectile is shown for the normal incident impact. In this field the velocity components of direction pointing radially outward were highest near the shear band within the pellet. Following the formation of the cup the plastic deformation and forward momentum of the projectile continues to drive the energetic material towards the impact face as well as radially outward, placing the sample under a high pressure loading state. This is important for later stages where the reaction is already taking place since this high pressure facilitates in the continued reaction of material. The continuous decrease in the size of the dark region at the center of the pellets footprint, observed in sapphire anvil tests, may be indicative of this mechanism. Furthermore, towards the center of the velocity field there is a stagnation region under high pressure and considerably less strain. In these types of experiments this can often result in small amount material bonded to the anvil face. In the case of tests conducted on AlFA, there was very little material adhered to the steel anvils from this process, which appeared to be consumed by the reaction, and was accompanied by scorch marks radiating from the center and diverging outward. Observations from sapphire anvil tests showed the initiation ring expanding radially. This is believed to be due to the combination of hot products igniting additional material and the induced radially straining of the pellet aided by the deformation of the copper projectile. The lack of unreacted material at the stagnation region suggests that the propagation of hot 
products may play a more dominant role in the reaction front expansion by overcoming radially induced straining from the projectile and consuming material near the center. This would not be unreasonable given the deflagration speeds of $700+\mathrm{m} / \mathrm{s}$ for Al/PTFE mixtures [11].

In these tests the mass of the copper projectile adds inertia and kinetic energy and therefore greater deformation at these lower impact velocities that contributes to the reaction. At higher impact velocities or higher kinetic energies, similar deformation behavior may likely occur in tests that instead uses a sabot which separates from the reactive material while in flight. This would put the reactive material in an less confined state. Since impact initiation studies on PTFE/Al have shown these types of composites to react at velocities as low as $172 \mathrm{~m} / \mathrm{s}$ using a more traditional Taylor test setup [25], we would not expect to observe large differences in the reactivity AlFA composites. Nevertheless, future tests should explore the projectile density-impact velocity parameter space more fully to deconvolve the effects of added kinetic energy from the projectile. However, additional mechanisms of inelastic deformation such as shock induced heating of the material from void collapse is going to be operative and play a more important role at higher velocities.

The formation of shear bands is a known mechanism [4, $26,27]$ for impact initiation of energetic materials. While other methods of initiation in energetic materials such as pore collapse $[28,29]$ and frictional heating with grit $[6$, $30,31]$ are also possible, shear banding appears to be dominant impact initiation mechanism for the AlFA composite system in this study. Through our simulations we have shown the shear bands to take on ring and crescent structures that compare well with the experimentally determined ignition locations and path of preferential spreading within the sample at the impact face. This spreading is consistent with observations made by Balzer et al. in drop-weight studies on PETN and RDX explosives, which showed ignition points that were located at adiabatic shear bands that then spread preferentially along these bands [26]. They also found hot-spots to have durations of $\sim 10 \mu$ s and sizes on the order of $\sim 0.1-10 \mu \mathrm{m}$. Our setup probes much higher strain-rate regimes that decrease the time duration to $<1 \mu \mathrm{s}$. While we cannot definitively determine critical hot-spot size by these methods, it can be safely assumed that they are smaller than $0.1 \mu \mathrm{m}$ since AlFA is comprised of nano-sized aluminum $(80 \mathrm{~nm})$, in contrast to explosive mixtures with grain size distributions that are typically much larger $(>>1 \mu \mathrm{m})$.

The critical hot-spot size may be linked to the coalescence of multiple hot-spots within the shear bands and is potentially a highly important mechanism to consider (for example, see Fig. 10). However, these localized features would be linked to microstructural details, which cannot be evaluated directly using continuum-based models. Moreover, while the bulk temperatures in our simulations are sufficiently high within the shear bands to begin the decomposition process of AIFA, there are likely much higher temperatures in the sample due to the heterogeneity of these types of composites.

In Figs. 8 and 9 we observed multiple ignition points, on one side of the sample, that coalesce into a single reaction front along the shear band. Results from simulation suggest this may be due to off normal impacts. This remains unresolved, since we are not able to simultaneously image side-profile and head-on impacts, i.e. this may be an artifact of a stochastic ignition process rather than enhanced adiabatic shear on one side due to tilt. Future modified Taylor impact tests should consider adding diagnostics that measure projectile tilt by either photon Doppler velocimetry (PDV), or multiple cameras (or one camera with strategically placed mirrors) to image orthogonal projectile motions within the gas gun chamber. If the head-on impact is simultaneously imaged, this would allow pitch and yaw to be directly correlated to the observed impact initiation locations. Electrical shorting pin may also be used. However, due to the projectiles small diameter they may interfere with the impact and reaction. PDV would be less intrusive than shorting pins but could also be challenging because due to the low tilt velocities. The added benefits of including additional diagnostics would have to weighed against the main advantage of these tests, which is their low cost and high speed of repetition.

\section{Conclusions}

Sapphire and steel anvil modified Taylor impact tests were conducted to examine the mechanical induced reaction initiation in fluorinated acrylate polymer composites containing $50 \mathrm{wt} \%$ of nano-aluminum particles. From side profile images obtained using a traditional steel anvil, the reaction was found to initiate at a time corresponding to near full compaction of the pellet. From head-on images using a sapphire window, the reaction was observed to initiate at an intermediate radius on the impact face of the pellet and spreads to first form a crescent shaped footprint before consuming the rest of the sample. By using experimental and model Al-PTFE data from the literature, we formulated a set of Zerilli-Armstrong model parameters for the AlFA composite, which were used in ALE3D continuum simulations. From these 3-D simulations, the ignition point and the subsequent preferential spreading of the reaction front are most likely located within a shear band, which correlates well with experimental observations. Simulations show temperatures within the samples that are high enough to decompose the fluorinated polymer matrix, as well as relatively high strains within the pellet at 
the time of reaction observed in the modified Taylor impact experiments. From simulations there is also evidence that straining of the copper projectile aids in the continued deformation of the pellet and reaction through a shear facilitated reaction process.

Acknowledgments Funding for this work was made available by Air Force Research Laboratory, Materials and Manufacturing Directorate, from the Nanoscience and Technology STT Initiative in Nanoenergetics. This work was also performed, in part, under the auspices of the U.S. Department of Energy by Lawrence Livermore National Laboratory under Contract DE-AC52-07NA27344 (LLNLJRNL-676602).

\section{References}

1. Cai J, Walley S, Hunt R, Proud W, Nesterenko V, Meyers M (2008) High-strain, high-strain-rate flow and failure in PTFE/AL/ W granular composites. Mat Sci Eng A 472:308-315. doi:10. 1016/j.msea.2007.03.068

2. Koch E-C (2002) Metal-fluorocarbon-pyrolants: III. Development and application of magnesium/Teflon/Viton (MTV). Propell Explos Pyrot 27:262-266

3. Du S, Thadhani N (2009) Impact initiation of pressed Al-based intermetallic forming powder mixture compacts. In: Elert $\mathrm{M}$, Butler W, Furnish M, Anderson W, Proud W (eds) Shock compression of condensed matter 2009, Nashville, TN. AIP Melville, New York, pp 470-473

4. Aydelotte B, Thadhani N (2013) Mechanistic aspects of impact initiated reactions in explosively consolidated metal+aluminum powder mixtures. Mat Sci Eng A 570:164-171

5. Boyle V, Frey R, Bines A (1996) Parallel/oblique impact on thin explosive samples. AIP Conf Proc 370:819-822. doi:10.1063/1. 50609

6. Perry W, Gunderson J, Balkey M, Dickson P (2010) Impactinduced friction ignition of an explosive: Infrared observations and modeling. J Appl Phys 108:084902. doi:10.1063/1.3487932

7. Walley S, Balzer J, Proud W, Field J (2000) Response of thermites to dynamic high pressure and shear. Proc $\mathrm{R}$ Soc $\mathrm{A}$ 456:1483-1503

8. Crouse C, Pierce C, Spowart J (2012) Synthesis and reactivity of aluminized fluorinated acrylic (AlFA) nanocomposites. Combust Flame 159:3199-3207

9. Crouse C, Spowart J (2011) Dynamic behavior of reactive aluminum nanoparticle composite materials. In: Shock Compression of Condensed Matter 2011, Chicago, IL (Unpublished paper)

10. Crouse C, Spowart J, Pierce C, Hardenstein B (2015) Particulatebased reactive nanocomposites and methods of making and using the same, Patent: US 9120710 B1

11. Dolgoborodov A, Makhov M, Kolbanev I, Streletskii A, Fortov V (2005) Detonation in an aluminum-Teflon mixture. JETP Lett 81:311-314

12. Zerilli F, Armstrong R (2002) Thermal activation constitutive model for polymers applied to polytetrafluoroethylene. In: Furnish M, Thadhani N, Horie Y (eds) Shock Compression of Condensed Matter 2001, Atlanta, GA. AIP, New York, pp 657-660

13. Zerilli F, Armstrong R (2007) A constitutive equation for the dynamic deformation behavior of polymers. J Mater Sci 42:4562-4575
14. Jordan J, Siviour C, Foley J, Brown E (2007) Compressive properties of extruded polytetrafluoroethylene. Polymer 48:4184-4195

15. Casem D (2008) Mechanical response of an Al-PTFE composite to uniaxial compression over a range of strain rates and temperatures. Technical Report, ARL-TR-4560, Army Research Laboratory

16. Rae P, Dattelbaum D (2004) The properties of poly(tetrafluoroethylene) (PTFE) in compression. Polymer 45:7615-7625

17. Chen W, Lu F, Cheng M (2002) Tension and compression tests of two polymers under quasi-static sand dynamic loading. Polym Test 21:113-121

18. Richeton J, Ahzi S, Vecchio K, Jiang F, Adharapurapu R (2006) Influence of temperature and strain rate on the mechanical behavior of three amorphous polymers: Characterization and modeling of the compressive yield stress. Int J Solids Struct 43:2318-2335

19. Steinberg D (1991) Equation of state and strength properties of selected materials. Technical Report, UCRL-MA-106439, Lawrence Livermore National Laboratory

20. Reuss A (1929) Berechnung der Fliegrenze von Mischkristallen auf Grund der Plastizittsbedingung fr Einkristalle. J Appl Math Mech 9:49-58. doi:10.1002/zamm.19290090104

21. Voigt W (1887) Theoretische Studien ber die Elasticittsverhltnisse der Krystalle. Abh $\mathrm{Kgl}$ Ges Wiss Gttingen, Math Kl 34:3-51

22. Hare D, Managan R (2006) CALE EOS form 2 fits for high pressure fused silica Hugoniot data. Technical Report, UCRLTR-217957, Lawrence Livermore National Laboratory

23. Nichols III, A (2014) ALE3D User's Manual Version 4.22. Technical Report, LLNL-SM-650174, Lawrence Livermore National Laboratory

24. White B, Springer H (2015) Investigation of the effects of microstructure and binder properties on impact-induced fragmentation in explosives with meso-scale simulations. In: Fifteenth international detonation symposium, San Francisco, CA, pp 1086-1095

25. Mock W, Holt W (2006) Impact initiation of rods of pressed polytetraflouroethylene (PTFE) and aluminum powders. In: Shock Compression of Condensed Matter 2005, Baltimore, MD. AIP, New York, pp 1097-1100. doi:10.1063/1.2263514

26. Balzer J, Field J, Gifford M, Proud W, Walley S (2002) Highspeed photographic study of the drop-weight impact response of ultrafine and conventional PETN and RDX. Combust Flame 130:298-306

27. Balzer J, Proud W, Walley S, Field J (2003) High-speed photographic study of the drop-weight impact response of RDX/DOS mixtures. Combust Flame 135:547-555

28. Field J (1992) Hot spot ignition mechanisms for explosives. Acc Chem Res 25:489-496. doi:10.1021/ar00023a002

29. Austin R, Barton N, Reaugh J, Fried L (2015) Direct numerical simulation of shear localization and decomposition reactions in shock-loaded HMX crystal. J Appl Phys 117:185902. doi:10. $1063 / 1.4918538$

30. Bowden F, Gurton O (1948) Initiation of explosions by grit particles. Nature 162:654-655

31. Dickson P, Parker G, Novak A (2010) Frictionally induced ignition processes in drop and skid tests. Fourteenth international detonation symposium. Coeur d'Alene, ID, pp 952-959 